\title{
Continuous Monitoring of Crosswell Seismic Travel Time
}

Thomas M. Daley* Lawrence Berkeley National Laboratory, Paul G. Silver, Carnegie Institute, Fenglin Niu, Rice University, Ernest L. Majer, Lawrence Berkeley National Laboratory

\section{Summary}

In two separate shallow field experiments, at two distance scales, we have used continuous monitoring to estimate the effect of barometric pressure on crosswell travel time and thereby calibrated the stress sensitivity of the rock volume between the wells. In a $3 \mathrm{~m}$ experiment we found a stress sensitivity of $10^{-6} / \mathrm{Pa}$ while in a $30 \mathrm{~m}$ experiment the sensitivity was $5 \times 10^{-8} / \mathrm{Pa}$. Results from a deeper $(1 \mathrm{~km})$, 2 month experiment at the San Andreas fault observation boreholes will be presented if analysis is completed.

\section{Introduction}

Measuring and monitoring changes in the subsurface stress field has been a long-sought goal of the geophysical community over recent decades. Continuous monitoring of crosswell seismic travel time has the potential to provide high-precision in-situ measurement of stress-induced velocity changes. An important component of in-situ monitoring of stress with active-source seismic measurement is calibrating the change in seismic property (e.g. P- or S-wave velocity) with a known stress variation, such as earth tides or atmospheric pressure change. Changes in seismic velocity induced by solid earth tidal loading and variations in barometric pressure have been estimated in some studies. The fractional change in seismic velocity with respect to stress change is reported to be in the range of $10^{-9} / \mathrm{Pa}$ to $10^{-6} / \mathrm{Pa}$. Recently, compelling observations of velocity change over a fixed path due to tides or barometric pressure have been made using underground emplaced instruments [Yamamura et al., 2003, and Sano, personal communication]. A logical next step is to take such measurements to field sites and use equipment which is designed for temporary deployment in wells of opportunity, allowing in-situ monitoring of stress response to asses the sensitivity. In this paper we present results from in-situ travel time monitoring using shallow $(10-100 \mathrm{~m})$ field site boreholes and equipment designed for crosswell seismic acquisition, recorded for time periods of 6 and 35 days.

\section{Measurability Theory}

Using a fixed source-receiver distance, we can measure the fractional change of seismic velocity, $\delta \hat{v}$, by measuring the delay time, $\tau$, between two seismograms with travel time $T$, i.e., $\delta \hat{v}=\tau / T$. We can express both $\tau$, and $T$, in terms of the characteristic frequency, $f_{0}$, $\tau=\varepsilon / f_{0}, \quad T=N / f_{0}$, where $N$ is the number of wavelengths between the source and receiver and $\varepsilon$ is the change in travel time per period, and then the velocity perturbation, $\delta \hat{v}$, can be rewritten as the ratio of two dimensionless parameters:

$$
\delta \hat{v}=\varepsilon / N
$$

From equation (1), it is clear that achieving the lowest possible detection threshold for $\delta \hat{v}$ can be broken into two separate tasks: 1) maximizing the number $N$ of wavelengths between source and receiver, and 2) increasing the precision in measuring normalized delay time, $\varepsilon$. The first task, maximizing $N$, is a function of the medium velocity, the path length $\mathrm{L}$, and the frequency, since $N$ can be written as $N=L f_{0} / V$. Thus, the acquisition geometry (path length) and maximum frequency (a function of the source and the medium's attenuation), will factor into maximizing $N$. For our experiments, values of $N$ range from 10 to about 50 . For the second task, there exists a theoretical lower bound which places a limit on the performance of all unbiased time delay estimators. This limit is known as the CramerRao Lower Bound (CRLB) [e.g., Carter, 1987; Walker and Trahey, 1995]. It predicts the standard deviation of delay time errors, $\sigma_{\tau}:$, where

$$
\sigma_{\tau} \geq \sqrt{\frac{3}{2 f_{0}^{3} \pi^{2} T\left(B^{3}+12 B\right)}\left(\frac{1}{\rho^{2}}\left(1+\frac{1}{S N R^{2}}\right)^{2}-1\right)}
$$

when two seismograms are relatively similar. Here, $f_{0}, B$, $\rho, S N R, T$ are the center frequency, fractional bandwidth, source waveform correlation coefficient, signal-to-noise ratio and kernel window length, respectively. In our case, $B$ $<1, \rho \approx 1, S N R>>1$, so that equation (2) can further simplified. Expressing the uncertainty in terms of $\varepsilon$,

$$
\sigma_{\varepsilon}=\sigma_{\tau} f_{0} \geq \frac{1}{2 \pi \cdot S N R} \sqrt{\frac{1}{f_{0} T B}} \sim \frac{1}{2 \pi \cdot S N R},
$$

since $\left(f_{o} T B\right)^{1 / 2}$ is of order unity in our application. Equation (3) makes two very important points. First, the SNR is the parameter that controls the relative uncertainty. For example, the SNRs for a single record and for 100 stacked records from the first test site are $\sim 300$ and $\sim 3000$, respectively. The resulting predicted precision is on the order of $10^{-3}$ and $10^{-4}$. These values correspond to errors of $10^{-7} \mathrm{~s}$ and $10^{-8} \mathrm{~s}$ in the delay time estimates, which will be shown to be in good agreement with our measurements. Second, the precision is not affected by sampling rate, as long as the digitizing error is much smaller than other sources of noise. This provides the theoretical basis for achieving sub-sample precision through interpolation. There are several methods that allow us to measure the 


\section{Continuous Crosswell Monitoring}

delay time to sub-sample precision. We have tested three different cross-correlation-based algorithms: (1) cosine fitting, (2) seismogram interpolation, and (3) crosscorrelation interpolation seismograms. In calculating the delay time between seismograms with high SNRs, all three algorithms yielded the same standard deviation of the mean delay time, of order $10^{-9} \sim 10^{-8} \mathrm{~s}$. We use algorithm (2), in which we calculate the Fourier transform of a time series, pad the frequency spectrum with zeros, and calculate the inverse Fourier transform. The data are interpolated to a time interval of $10^{-8} \mathrm{~s}$. We then calculate the cross correlation. We have extensively stacked the data and investigated whether the $\mathrm{N}^{1 / 2}$ rule works for our case. Massive stacking (10 per second) allows us to obtain a SNR of $10^{4}-10^{5}$ for one-hour intervals, corresponding to $\varepsilon$ on the order of $10^{-5}$ and $10^{-6}$ and detectability of $\delta \hat{v}$ on the order of $10^{-6}$ and $10^{-7}$.

The CRLB expression (3) can be used to determine a characteristic frequency or alternatively a measurement distance that optimizes SNR. By the definition of attenuation, signal amplitude is proportional to $(1-\pi / Q){ }^{N}$. Using (3), then, the standard deviation of $\varepsilon / \mathrm{N}$ is proportional to $(1-\pi / \mathrm{Q})^{-\mathrm{N}} \mathrm{N}^{-1}$. One can then find an optimal value for $\mathrm{N}$ that minimizes $\varepsilon / \mathrm{N}$ by differentiating this expression with respect to $\mathrm{N}$ and setting it equal to zero. For $\pi / \mathrm{Q}<<1$, we find $N \sim Q / \pi$. Thus, there is an optimal value of $\mathrm{N}$ that gives an optimal frequency band for every distance. For example, if $\mathrm{Q}=60(\mathrm{~N}=20)$ and assuming the $\mathrm{P}$ velocity is $1.5 \mathrm{~km} / \mathrm{s}$, the optimal frequencies for sourcereceiver distances of $3 \mathrm{~m}, 30 \mathrm{~m}, 100 \mathrm{~m}$ and $1000 \mathrm{~m}$ (corresponding to the distance range that we expect to encounter) are $10 \mathrm{KHz}, 1.0 \mathrm{KHz}, 330 \mathrm{~Hz}$, and $33 \mathrm{~Hz}$, respectively. For our two test sites we were able to use 10 $\mathrm{kHz}$ and $1 \mathrm{kHz}$ at 3 and $30 \mathrm{~m}$, respectively. The analysis at our two test sites shows that we can achieve $\varepsilon$ of about $10^{-5}$ for a one-hour measurement interval. Thus we expect the detectable $\delta \hat{v}$ to be of order $10^{-6}$.

\section{Method}

Data acquisition for continuous crosswell monitoring builds on development of crosswell seismic work of the previous 10-20 years. Our acquisition was conducted with a combination of commercial and special-built equipment. The special-built components are the piezoelectric source and the high voltage amplifier used to power it. The source is cylindrical rings of piezoelectric ceramic epoxied together and wired for positive and negative voltage on the inner and outer surfaces. The sensors were commercial hydrophones designed for large bandwidth and high sensitivity using built-in amplifiers with analog data transmission. The commercial recording system has a 24 bit analog-to-digital converter using a sigma-delta conversion algorithm. Our data was collected at a sampling rate of 48,000 samples per second. The start time is computed to within a $20^{\text {th }}$ of a sample. Thus there is a delay time measurement error that will be a $40^{\text {th }}$ of a sample (half-way between samples), and the average error will be an $80^{\text {th }}$ of a sample, assuming that the errors are uniformly distributed. So this corresponds to an average error of $260 \mathrm{~ns}$ per trigger. The error in the stacked data is smaller by a factor of $\mathrm{N}^{1 / 2}$, assuming they are uncorrelated. For $\mathrm{N}=36,000$ traces, as in our experiments, the error is $1.4 \mathrm{~ns}$ for one-hour sampling, which is below other sources of error.

\section{Field Experiments}

Our first test was conducted at an interwell distance of about $3 \mathrm{~m}$ between two $15-\mathrm{m}$ deep holes at the Lawrence Berkeley National Laboratory (LBNL). The sensor cable had 24 sensors at $0.5 \mathrm{~m}$ spacing. The source was pulsed every $100 \mathrm{~ms}$ with a record length of 10 milliseconds. These traces, with travel time of about $2 \mathrm{~ms}$ were stacked in the acquisition system with output of a stack of 600 single traces each minute (Figure 1a). We continuously recorded a total of 160 hours in November 2003. Figure 1b shows that SNR for this experiment continues to increase for up to $10^{4}$ stacks, implying that a non-random noise 'floor' has not been reached. The SNR average was about 4000 for the stacked records. The best achievable precision of $\varepsilon$, based on equation (3) is $10^{-5} \sim 10^{-4}$. Since the center frequency of the data $f_{0}$ is $10 \mathrm{KHz}$, the corresponding best achievable precision in delay time, $\sigma_{\tau}$ : , is $10^{-9} \sim 10^{-8} \mathrm{~s}$, i.e. a few ns. Figure 1c shows a histogram of the measured delay time between two adjacent one-minute records, which follows a normal distribution with a standard deviation of $\sim 50 \mathrm{~ns}$, about an order of magnitude larger than the CRLB. For one-hour stacking, we can achieve a standard deviation, or precision, of up to $6 \mathrm{~ns}$, which corresponds to a resolution of $3 \times 10^{-6}$ on the velocity perturbation, $\delta \hat{v}$, in good agreement with the previously stated uncertainty expectation of $10^{-6}$.

In this experiment we measured the delay time using a time window which contained both the first arrival and coda (10 cycles). During the period of observation, we see a large pressure excursion $\left(\sim 10^{3} \mathrm{~Pa}\right)$ which continues for 3 days (Figure 2). It is clear that the delay times track this barometric pressure change. Using the variations in delay time and the barometric pressure change, we obtain a value of stress sensitivity of $10^{-6} / \mathrm{Pa}$.

The sign of the travel time fluctuation relative to the barometric pressure (increasing travel time for increasing pressure) is the opposite of standard expectation and requires some explanation. Under dry conditions, we 


\section{Continuous Crosswell Monitoring}

expect increased barometric pressure to correspond to decreased travel time. The increased barometric load serves to close cracks, thus reducing crack density and increasing seismic velocity. The source and receivers are in water-filled boreholes that are likely in communication with the local aquifer allowing large pore pressure changes near the borehole. Under these conditions, it is possible to get either a positive or negative response to barometric pressure, depending on the barometric efficiency and the spacing between the source and receiver. A measurement in the near-field of a fluid-filled borehole in porous, permeable material (such as our $3 \mathrm{~m} \mathrm{LBNL}$ test) could measure effective stresses of opposite sign to far field (barometric or tidal) stress. Qualitatively, this provides a reasonable explanation for our initial observations.

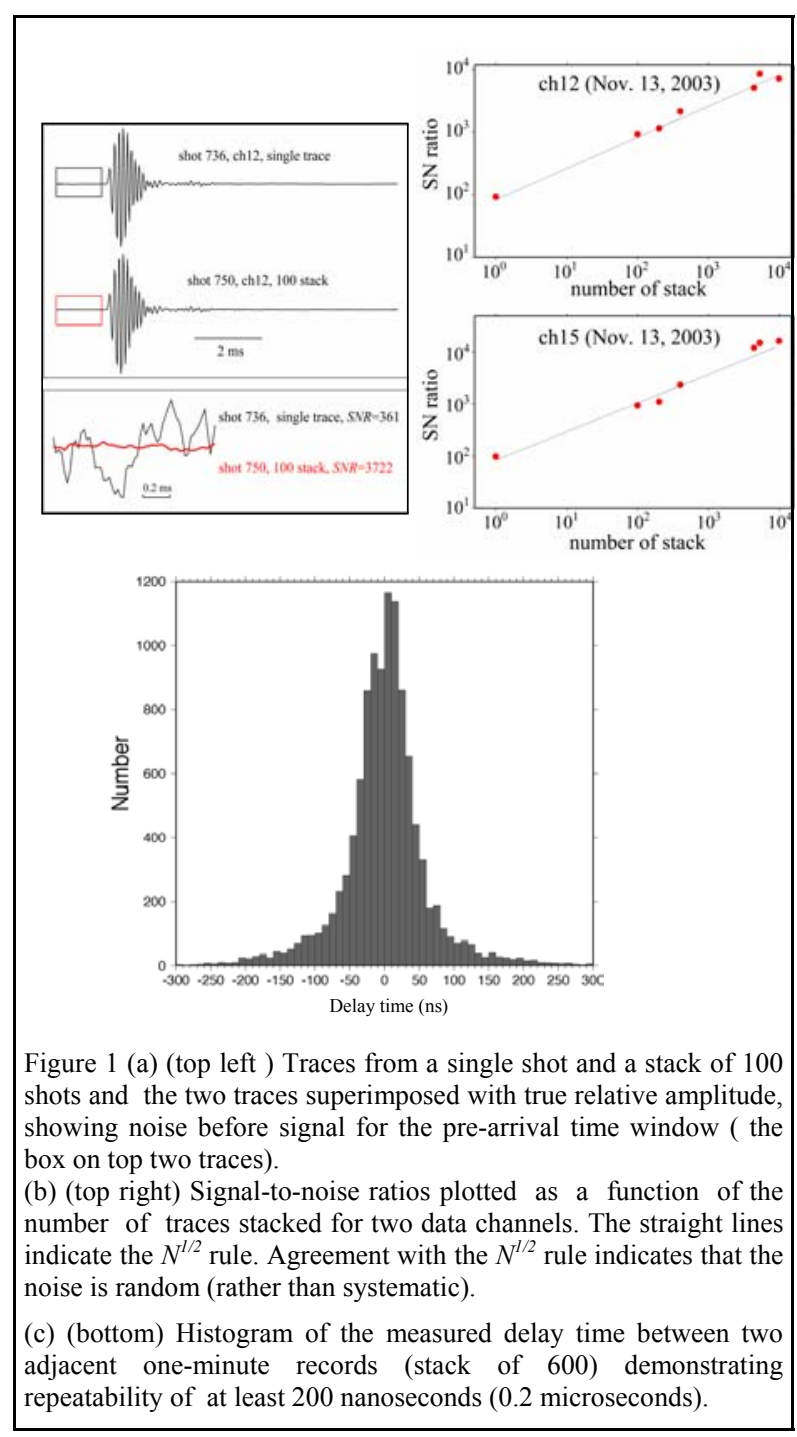

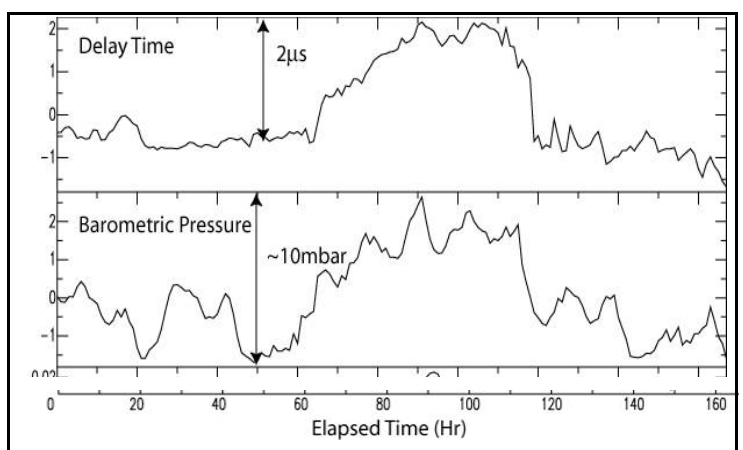

Figure 2: Delay times for the LBNL test site derived from the first time plus the coda (top) with barometric pressure change (bottom). Maximum change of 2 microseconds corresponds with 10 millibar.

After the LBNL site work, we deployed in deeper wells and at a longer interwell distance, hopefully moving out of the near-well conditions at the LBNL site. We chose to deploy in wells at the Richmond (California) Field Station (RFS) test site. The RFS test site has several boreholes approximately $70 \mathrm{~m}$ deep and $30 \mathrm{~m}$ apart from each other. A source was set $25 \mathrm{~m}$ below surface, and the 24-element hydrophone array was placed at the depth range 25-36.5 m below surface.

The source was repeated 10 times per second, with a $50 \mathrm{~ms}$ record length, and these pulses were stacked by the recording system to give recordings every 1 minute (600 pulses). For this 35 day long study, we attempted to reduce any movement of the sensors with 'centralizers' (flexible bands of metal acting like springs) which kept 3 of the sensors fixed, and with hard foam attached to the source. However, at some point during deployment the source foam was compressed by the water pressure. The dominant frequency observed in the data was about $1 \mathrm{kHz}$ and the travel time was about $20 \mathrm{~ms}$. Compared to the LBNL test, the data frequency is about $1 / 10$, but the travel time is 10

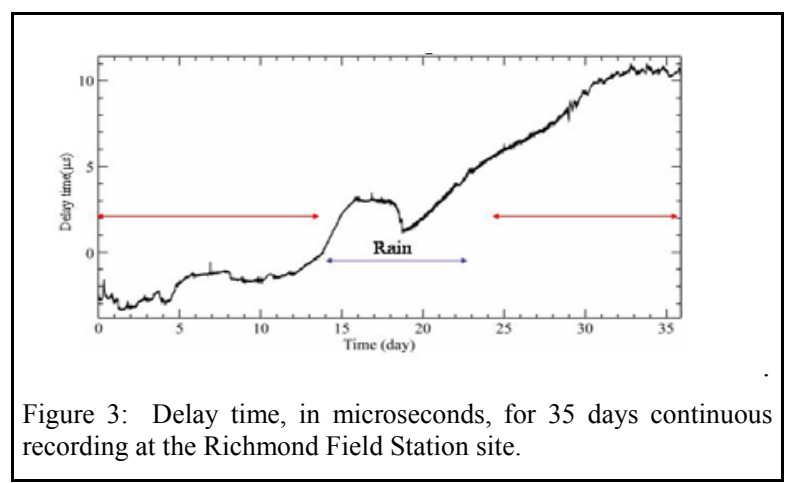




\section{Continuous Crosswell Monitoring}

times as large, therefore we achieve a resolution for $\delta \hat{v}$ that is essentially the same as the value we obtained at LBNL, $\sim 3 \times 10^{-6}$.) The SNR of 1 minute stacks was 604 with a standard deviation of $39.2 \mathrm{~ns}$, while 1 hour stacks had a SNR of 3185 with 5.2 ns standard deviation.

We observed that delay time correlates very strongly with temperature as recorded inside the recording container. The scaling is about $0.1 \mathrm{us} /{ }^{\circ} \mathrm{C}$ where swings of $10^{\circ} \mathrm{C}$ correspond to oscillations in delay time of about a microsecond A linear scaling constant between the measured temperature and the delay time variation was estimated. The scaled temperature data was then subtracted from the delay time data. We also minimized the effect by maintaining the electronics within a temperature range, which was done midway through the experiment.

The data collection had a period of heavy rain as indicated in Figure 3, and we found that data from the rainfall period is problematic to interpret. At least two possible effects are involved, an increase in surface load (increasing effective stress) due to the mass of the rain water, and an increase in pore pressure (decreasing effective stress) due to the infiltration of rain water. The effect on travel time due to rain is therefore difficult to interpret and we do not do so. We also have removed the linear trend seen in Figure 3 before analysis. The cause of the trend is unknown, but is possibly cable stretch.

Figure 4 shows the delay time and barometric pressure for two time periods, before and after the rain,

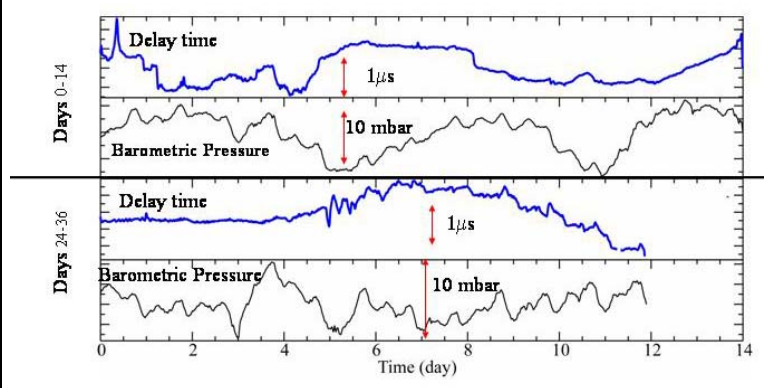

Figure 4: Delay time and barometric pressure for the RFS site for pre rain days 0-14 (top) and post rain days 24-36 (bottom).

corresponding to days 1-14 and 24-36. The correspondence of time delay to pressure is not entirely consistent, however in both time periods there is a long period (4-8 days) correspondence with a delay time change of 1 microsecond coincident with a barometric pressure change of 10 millibar. The data had a full scale variation of $\delta \hat{v}=5 \times 10^{-5}$, giving a stress sensitivity $5 \times 10^{-8} / \mathrm{Pa}$. Importantly, the sign of travel time fluctuation relative to the barometric pressure is now the expected decrease in travel time for increasing pressure, indicating that we are measuring the 'far field' effect away from the boreholes

\section{Conclusions}

The state-of-the-art in controlled source seismic acquisition has advanced to the point of measuring microsecond variations in travel time, allowing in-situ measurement of subsurface stress via the stress sensitivity of seismic velocity. The crosswell acquisition geometry provides access to the subsurface without the large variability associated with near-surface measurement. Importantly, natural stress signals such as barometric pressure or solid earth tides provide the means to calibrate the stress sensitivity of a given rock volume. At the $3 \mathrm{~m}$ scale, the stress sensitivity appears to be controlled by near-well pore fluid pressure increase which decreases effective stress in the rock for increasing barometric pressure. At the $30 \mathrm{~m}$ scale, travel time measurement is in a 'far field' region in which pore pressure is stable and increasing barometric pressure increases the effective stress. Quantitative analysis of the crossover between these two regimes requires a coupled hydro-mechanical model of the subsurface. The type of calibration measurements shown here provide the foundation for interpretation of any long term measurement of stress change, such as that preceding earthquakes, or that due to fluid injection or withdrawal. While these temporary, cable deployed measurements have remarkable repeatability, permanent emplacement of borehole sources and sensors allows increased accuracy in delay-time and stress-sensitivity estimation.

\section{References}

Carter, G.C., Coherence and time delay estimation, 1987, Proc. IEEE, 75, 236-255.

Walker W.F., and G.E. Trahey, 1995, Limits on the performance of near field phase aberration correction, IEEE Ultrasonic Symposium, 1461-1465.

Yamamura, K., O. Sano, H. Utada, Y. Takei, S. Nakao, and Y. Fukao, 2003, Long-term observation of in situ seismic velocity and attenuation, Journal of Geophysical Research, 108, 10.1029/2002JB002005.

\section{Acknowledgments}

This work was supported by the National Science Foundation (authors Silver and Niu) and by the U.S. Dept. of Energy, Lawrence Berkeley National Laboratory, Earth Science Division. 


\section{EDITED REFERENCES}

Note: This reference list is a copy-edited version of the reference list submitted by the author. Reference lists for the 2006 SEG Technical Program Expanded Abstracts have been copy edited so that references provided with the online metadata for each paper will achieve a high degree of linking to cited sources that appear on the Web.

\section{REFERENCES}

Cole, K. S., and R. H. Cole, 1941, Dispersion and absorption in dielectrics: Journal of Carter, G.C., Coherence and time delay estimation, 1987: Proceedings of the IEEE, 75, 236-255.

Walker W.F., and G.E. Trahey, 1995, Limits on the performance of near field phase aberration correction: IEEE Ultrasonic Symposium, 1461-1465.

Yamamura, K., O. Sano, H. Utada, Y. Takei, S. Nakao, and Y. Fukao, 2003, Long-term observation of in situ seismic velocity and attenuation: Journal of Geophysical Research, 108, 10.1029/2002JB002005. 